\title{
The topology of covert conflict
}

\author{
Shishir Nagaraja, Ross Anderson \\ Computer Laboratory \\ JJ Thomson Avenue, Cambridge CB3 0FD, UK \\ forename.surname @ cl.cam.ac.uk
}

\begin{abstract}
Often an attacker tries to disconnect a network by destroying nodes or edges, while the defender counters using various resilience mechanisms. Examples include a music industry body attempting to close down a peer-to-peer file-sharing network; medics attempting to halt the spread of an infectious disease by selective vaccination; and a police agency trying to decapitate a terrorist organisation. Albert, Jeong and Barabási famously analysed the static case, and showed that vertex

-order attacks are effective against scale-free networks. We extend this work to the dynamic case by developing a framework based on evolutionary game theory to explore the interaction of attack and defence strategies. We show, first, that naive defences don't work against vertex-order attack; second, that defences based on simple redundancy don't work much better, but that defences based on cliques work well; third, that attacks based on centrality work better against clique defences than vertex-order attacks do; and fourth, that defences based on complex strategies such as delegation plus clique resist centrality attacks better than simple clique defences. Our models thus build a bridge between network analysis and evolutionary game theory, and provide a framework for analysing defence and attack in networks where topology matters. They suggest definitions of efficiency of attack and defence, and may even explain the evolution of insurgent organisations from networks of cells to a more virtual leadership that facilitates operations rather than directing them. Finally, we draw some conclusions and present possible directions for future research.
\end{abstract}

\section{Introduction}

Many modern conflicts turn on connectivity. In conventional war, much effort is expended on disrupting the other side's command, control and communications by jamming or destroying his facilities. Counterterrorism operations involve a similar effort but with different tools: traffic analysis to trace communications, coupled with surveillance of the flows of money, material and recruits, followed by the arrest and interrogation of individuals who appear to be significant nodes. Terrorists are aware of this, and take measures to prevent their networks being traced. Usama bin Laden described his strategy on the videotape captured in Afghanistan as 'Those who were trained to fly didn't know the others. One group of people didn't know the other group' (see 14, which describes the hijackers' networks).

Connectivity matters for social dominance too, as a handful of leading individuals do much of the work of holding a society together. Subverting or killing these leaders is likely to be the cheapest way to make an invaded country submit. When the Norman French invaded England in the eleventh century, they killed or impoverished most of the indigenous landowners; when the Turks, and then the Mongols, invaded India, they killed both landowners and priests; when England suppressed the Scottish highlands after the 1745 uprising, landowners were induced to move to Edinburgh or London; and in many of the dreadful events of the last century, rulers targeted the elite (Russian kulaks, Polish officers, Tutsi schoolteachers, ... ).

Moving from politics to commerce, the music industry spends a lot of money attempting to disrupt peer-to-peer file-sharing networks. Techniques range from technical attacks to aggressive litigation against individuals believed to have been running major nodes.

Networks of personal contacts are important in other applications too. In public 
health, for example, it often happens that a small number of individuals account for much of the transmission of a disease. Thus Senegal has been more effective at tackling the spread of HIV/AIDS than other African countries, as they targeted prostitutes [19]. In fact, interest in social networks has grown greatly over the last 15 years in the humanities and social sciences 209.

Recent advances in the theory of networks have provided us with the mathematical and computational tools to understand such phenomena better. One striking result is that a network much of whose connectivity comes from a small number of highly-connected nodes can be very efficient, but at the cost of extreme vulnerability. As a simple example, if everyone in the county communicates using one telephone exchange, and that burns down, then everyone is isolated.

This paper starts to explore the tactical and strategic options open to combatants in such conflicts. What strategies can one adopt, when building a network, to provide good trade-offs between efficiency and resilience? We are particularly interested in complex networks, involving thousands or millions of nodes, which are so complicated (or under such dispersed control) that the resilience rules can only be implemented locally, rather than by a central planner who deliberately designs a network with multiple redundant backbones.

Is it possible, for example, to create a virtual high-degree node, by combining a number of nodes which appear on external inspection to have lower degree? For example, a number of individuals might join together in a ring, and use some covert communications channel to route sensitive information round the ring in a manner shielded from casual external inspection. There is a loose precedent in Chaum's 'dining cryptographers' construction 10, in which a number of cryptographers pass messages round a ring in such a way as to mask, from insiders, the source and destination of encrypted traffic. Can we build a similar construction, but in which the fact of systematic message routing is concealed from outsiders, with the result that the participants appear to be 'ordinary' nodes making a modest contribution in the network, rather than important nodes that should be targeted for close inspection and/or destruction?

\section{Previous Work}

There has been rapid progress in recent years in understanding how networks can develop organically, how their growth influences their topology, and how the topology in turn affects both their capacity and their robustness. There is now a substantial literature: for a book-length introduction, see Watts [21, while literature surveys are 117.

Early work by Erdös and Renyi modelled networks as random graphs [117]; this is mathematically interesting but does not model most real-world networks accurately. In real networks, path lengths are generally shorter; it is well known that any two people are linked by a chain of maybe half a dozen others who are pairwise acquainted - known as the 'small-world' phenomenon. This idea was popularised by Milgram in the 60s [16]. An explanation started to emerge in 1998 when Watts and Strogatz produced the alpha model. Alpha is a parameter that expresses the tendency of nodes to introduce their neighbours to each other; with $\alpha=0$, each node is connected to its neighbours' neighbours, so the network is a set of disconnected cliques, while with $\alpha=\infty$, we have a random graph. They discovered that, for critical values of $\alpha$, a small-world network resulted. The alpha model is rather complex to analyse, so they next introduced the beta network: this is constructed by arranging nodes in a ring, each node being connected to its $r$ neighbours on either side, then replacing existing links with random links according to a parameter $\beta$; for $\beta=0$ no links are replaced, and for $\beta=1$ all links have been replaced, so that the network has again become a random graph 22. The effect is to provide a mix of local and long-distance links that models observed phenomena in social and other networks. 
How do networks with short path lengths come about in the real world? The simplest explanation involves preferential attachment. Barabási and Albert showed in 1999 how, if new nodes in a network prefer to attach to nodes that already have many edges, this leads to a power-law distribution of vertex order which in turn gives rise to a scale-free network [6], which turns out to be a more common type of network than the alpha or beta types. In a social network, for example, people who already have many friends are useful to know, so their friendship is particularly sought by newcomers. In friendship terms, the rich get richer. There are many economic contexts in which such dynamics are also of interest [13.

The key paper for our purposes was written by Albert, Jeong, and Barabási in 2000. They observed that the connectivity of scale-free networks, which depends on the highly-connected nodes, comes at a price: the destruction of these nodes will disconnect the network. If an attacker removes the best-connected nodes one after another, then past some threshold point the size of the largest component of the graph collapses 2].

Later work by Holme, Kim, Yoon and Han in 2002 extended this from attacks on vertices to attacks on edges; here, the attacker removes edges connecting high-degree nodes, and again, past some critical point, the network becomes disconnected [15]. They also suggested using centrality - technically, this is the 'betweenness centrality' of Freeman [12 - as an alternative to degree for attack targeting. (A node's centrality is, roughly speaking, the proportion of paths on which it lies.) Computing centrality is harder work for the attacker than observing vertex degree, but it enables him to attack networks (such as beta networks) where there is little or no variability in vertex order. Finally, in 2004, Zhao, Park and Lai modelled the circumstances in which a scale-free network can suffer cascading breakdown from the successive failure of high-connectivity nodes 23. These ideas find some resonance in the field of strategic studies: for example, Soviet doctrine called for destroying a third of the enemy's network, jamming a further third, and hoping that the remaining third would collapse under the increased weight of traffic.

\section{Naive Defences Don't Work}

Given the obvious importance of the subject, and the fact that the Albert-Jeong-Barabási paper appeared in 2000, one obvious question is why there has been no published work since on how a network can defend itself against a decapitation attack. Here is one possible explanation: the two obvious defences don't work.

One of these is simply to replenish destroyed nodes with new nodes, and furnish them with edges according to the same scale-free rule that was used to generate the network initially. One might hope that some equilibrium would be found between attack and defence.

The other obvious defence is to replenish destroyed nodes, but to wire their edges according to a random graph model. In this way, we might hope that, under attack, a network would evolve from an efficient scale-free structure into a less efficient but more resilient random structure. In a real application, this might happen either as a result of nodes learning new behaviour, or by selective pressure on a node population with heterogeneous connectivity preferences: in peacetime the nodes with higher degree would become hubs, while in wartime they would be early casualties.

Nice as these ideas may seem in theory, they do not work at all well in practice. Figure [1 shows first (solid line) how the vertex-order attack of Albert, Jeong and Barabási works against a simulated network with no replenishment, then with random replenishment, then with scalefree replenishment. In the vanilla case the attack takes two rounds to disconnect the network; with random replenishment it takes three, and with scale-free replenishment it takes four.

It seems that, to defend against these kinds of decapitation attacks on networks, we will need smarter defence strategies. But how should these be evolved, and what sort of framework should we use to evaluate them? 


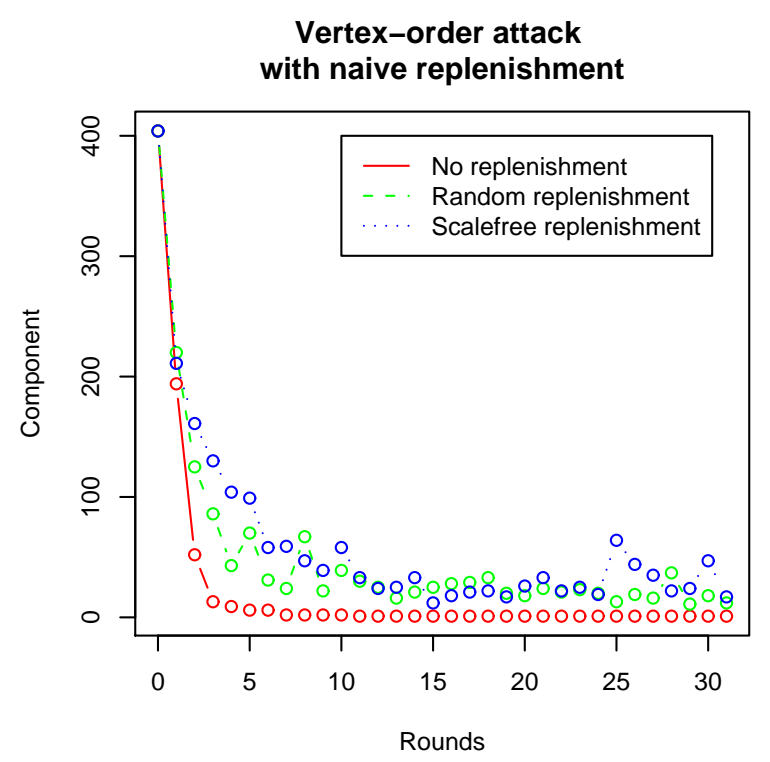

$\begin{array}{ll} & \text { No-replenishment } \\ \text { Ra___........ } & \text { Random-replenishment } \\ \text { Scalefree-replenishment }\end{array}$

Fig. 1. Naive defences against vertex-order decapitation attack

\section{A Model from Evolutionary Game Theory}

Previous researchers considered disruptive attacks on networks to be a single-round game. Such a model is suitable for applications such as a conventional war, in which the attacker has to expend a certain amount of effort to destroy the defender's command, control and communications, and one wishes to estimate how much; or a single epidemic in which a certain amount of resource must be spent to bring the disease under control.

However, there are many applications in which attack and defense evolve through multiple rounds: terrorism and music-sharing are only two examples. We now develop a framework for considering this more general case. We apply ideas from evolutionary game theory developed by Axelrod and others 34. This theory studies how games of multiple rounds differ from single-round games, and it has turned out to have significant explanatory power in applications from ethology to economics.
We now formalise a model in which a game is played with a number of rounds. Each round consists of attack followed by recovery. Recovery in turn consists of two phases: replenishment and adaptation.

In the attack phase, the attacker destroys a number of nodes (or, in a variant, of edges); this number is his budget. He selects nodes for destruction according to some rule, which is his strategy. For example, he might at each round destroy the ten nodes with the largest number of edges connected to them. He executes this strategy on the basis of information about the network topology.

In the replenishment phase, the defending nodes recruit a number of new nodes, and go through a phase of establishing connections - again, according to given strategies and information.

In the adaptation phase, the defending nodes may rewire links within each connected component of the network, in accordance with some defensive strategy. The adaptation phase is applied once at the start of the game, before the first round of attack; thereafter the game proceeds attack - replenish - adapt.

An attack strategy is more efficient, for a given defense strategy, if an attacker using it requires a smaller budget to disrupt the network. Similarly, a defense strategy is more efficient if, for a given attack strategy, it compels the attacker to expend a higher budget to achieve network disruption. (We will clarify this later once we have presented and discussed a few simulations.)

We assume initially that the attacker has perfect information about the network topology, and that her goal is simply to partition the network - that is, divide it into two or more nontrivial disjoint components. We assume that the defender has only local information, that it, each node shares the information available to those nodes with which it is connected. Thus, for example, if the attacker manages to split the network into two components, there is no way for them to reconnect. We also start off by assuming that the defence strategy affects only the adaptation phase, as only once nodes have connected to a network can they be 
programmed to follow it; so the replenishment phase is exogenous.

A further initial assumption is that the attack and defence budgets are roughly equal. By this we will mean that for each node destroyed in the attack phase, one node will be replaced in the resource addition phase. Thus the network will neither grow or shrink in absolute size and we can concentrate on connectivity effects. We will discuss other possible assumptions later, but the static budgets and global attack / local defence assumptions will get us started.

\section{Defence Evolution - First Round}

To analyse the vulnerability of a network, the selection of network elements (nodes or edges) destroyed in each round is the attacker's choice and constitutes her strategy. The attacker wishes to maximize the network damage caused per unit of work.

We will start off by considering a static attacker, using what we know to be a reasonable attack (vertex-order), and examine how the defence strategy can adapt. Then we will see what better attacks can be found against the best defence we found. Then we will look for a defence against the best attack we found in the last round, and so on. There is no guarantee that the process converges - there may be a specialised attack that works well against each defence, and vice versa - but if evolutionary games on networks behave like more traditional evolutionary games, we may expect to find some strategies that do well overall, as 'tit for tat' does in multi-round prisoners' dilemma. We may also expect to gain useful insights in the process.

\subsection{Defense strategy 1 - random replenishment}

Our first defensive strategy is the simplest of all, and is one of the naive defences introduced in the above section. New nodes are joined to the graph at random. We assume that each attack round removes $r$ nodes, and the replenishment round adds exactly $r$ nodes, each of which is joined to the surviving vertices with probability $p . r$ remains constant for each run of the simulation, while $p$ increases from $k /(N-r)$ to $k /(N-1)$ as the replenishment proceeds. In this strategy, the defender does nothing in the adaptation phase.

This models the case where new recruits to a subversive network simply contact any other subversives they can find; no attempt is made to reshape the network in response to the capture of leaders but the network is simply allowed to become more amorphous.

\subsection{Defense strategy 2 - dining steganographers}

Our second defensive strategy is more sophisticated, and is inspired by the theory of anonymous communication as developed by computer scientists, most notably Chaum [10]. A node that acquires a high vertex order, and thus could be threatened by a vertex-order attack, splits itself into $n$ nodes, arranged in a ring. The rings have two functions. First, they provide resilience: a ring broken at one point still supports communications between all its surviving nodes, and it is the simplest such structure. Second, nodes can route covert traffic between appropriate input and output links, and use encryption and other information-hiding mechanisms to conceal the traffic. This model was originally presented in Chaum's seminal 'dining cryptographers' paper cited above, so we might refer to it as the 'dining steganographers'. The collaborating nodes in each ring cannot conceal the existence of communication between them, as the cover traffic is visible to the attacker. However, from the attacker's viewpoint it is not obvious that these $n$ nodes are acting as a virtual supernode.

Our focus here is on the effects of network topology, rather than on the higher-layer mechanisms that actually implement the covertness property and that provide any confidentiality of content or of routing data. We assume a world in which there is sufficient encrypted traffic (SSL, SSH, DRM, . . . ) that encrypted traffic is not of itself suspicious so long as it is wrapped in a common ciphertext type. The attacker's input consists of traffic data collected from the 
backbone or from ISPs, and her output consists of decisions to send police officers to raid the premises associated with particular IP addresses. Her problem is this: given an observed pattern of communications, whom should she investigate first?

The precise mechanism of ring formation in our simulation is as follows. A vulnerable node decides to create a ring and recruits for the purpose a further $n-1$ nodes from the new nodes introduced in the most recent replenishment round, or, if they are inadequate, from among its immediate neighbours. Existing ring members cannot be recruited, so rings may not overlap. Finally, recruits to a ring relinquish any existing links with the rest of the network, and the ring-forming node shares its external links uniformly among all the members of the ring.

\subsection{Defense strategy 3 - revolutionary cells}

Our third defensive strategy is inspired by cells of revolutionaries, along the model favoured historically by a number of insurgent organisations. A node that acquires a high vertex order splits itself into $n$ nodes, all linked with each other, with the previous outside connections split uniformly between them. In graph-theoretic language, each supernode is a clique.

As in ring formation, a node that considers itself vulnerable is allowed to split itself into a clique of nodes. The new nodes are drawn either from the pool of new nodes, or, if they are insufficient, from low-vertex-order neighbours of the clique-forming node. As before, this node's external edges are distributed uniformly among members, while other member nodes' former external edges are deleted.

Simulations - first set For our first set of simulations, we consider a scalefree network of $N=400$ nodes. We use a Barabási-Albert network created by the following algorithm:

1. Growth: Starting with $m_{0}=40$ nodes, at each round we add $m=10$ new nodes, each with 3 edges.
2. Preferential Attachment: The probability that a new node connects to node $i$ is $\Pi\left(k_{i}\right)=k_{i} / \sum_{j} k_{j}$ where $k_{i}$ is the degree of node $i$.

Having created the scalefree network, we then ran each of the above defensive strategies against a vertex-order attack.

Results The results of the initial three simulations are given in Figure 2

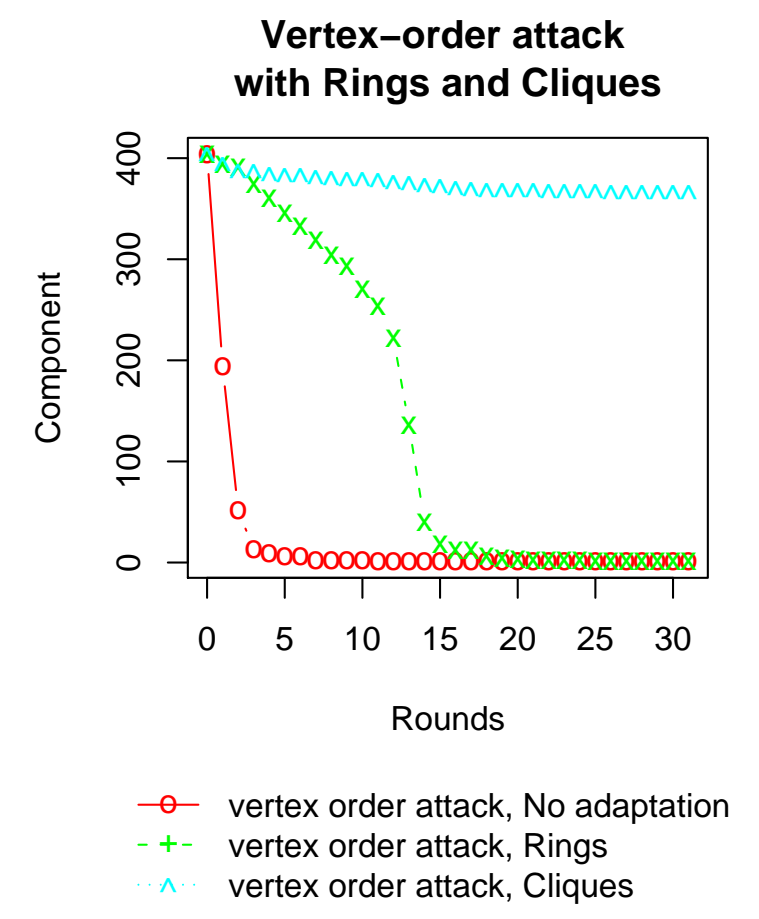

Fig. 2. Vertex order decapitation attack in rings, cliques and with no adaptation

The red graph in Figure 2 provides a calibration baseline. As seen in the above section, random replenishment without adaptation is ineffective: within three rounds the size of the largest connected component has fallen by a half, from 400 nodes to well under 200.

The green graph shows that rings give only a surprisingly short-term defence benefit. They postpone network collapse from about two rounds qto about a dozen rounds. Thereafter, the network is almost completely 
disconnected. In fact, the outcome is even worse than with random replenishment.

Cliques, on the other hand, work well. A few vertices are disconnected at each attack round, but as the cyan graph shows, the network itself remains robustly connected. This may provide some insight into why, although rings have seemed attractive to theoreticians, those real revolutionary movements that have left some trace in the history books have used a cell structure instead.

\section{$6 \quad$ Attack Evolution - First Round}

Having tried a number of defence strategies and found that one of them - cliques - is effective, the next step is to try out a number of attack strategies to see if any of them is effective against our defences, and in particular against cliques.

Of the attack strategies we tried against a clique defence, the best performer is an attack based on centrality. We used the centrality algorithm of Brandes [8] to select the highest-centrality nodes for destruction at each round. As before, our calibration baseline is random replenishment. For this, the red and black graphs show performance against vertex-order and centrality attacks respectively. Both are equally effective; within two or three rounds the size of the largest connected component has been halved.

The green and blue graphs show that the same holds for rings: the network collapses completely after about a dozen rounds. Centrality attacks are very slightly more effective but there is not much in it.

The most interesting results from these simulations come from the magenta and cyan graphs, which show how cliques behave. Cyan shows, as before, a vertex-order attack with severity $m=10$ being ineffective against a clique defence. Magenta shows the effect on such a network of a centrality attack. Here the largest connected component retains about 400 nodes until the network suddenly partitions at 14 rounds, whereafter a largest-component size of about 200 is maintained stably.

\section{Vertex-order and Centrality attack with Rings and Cliques}

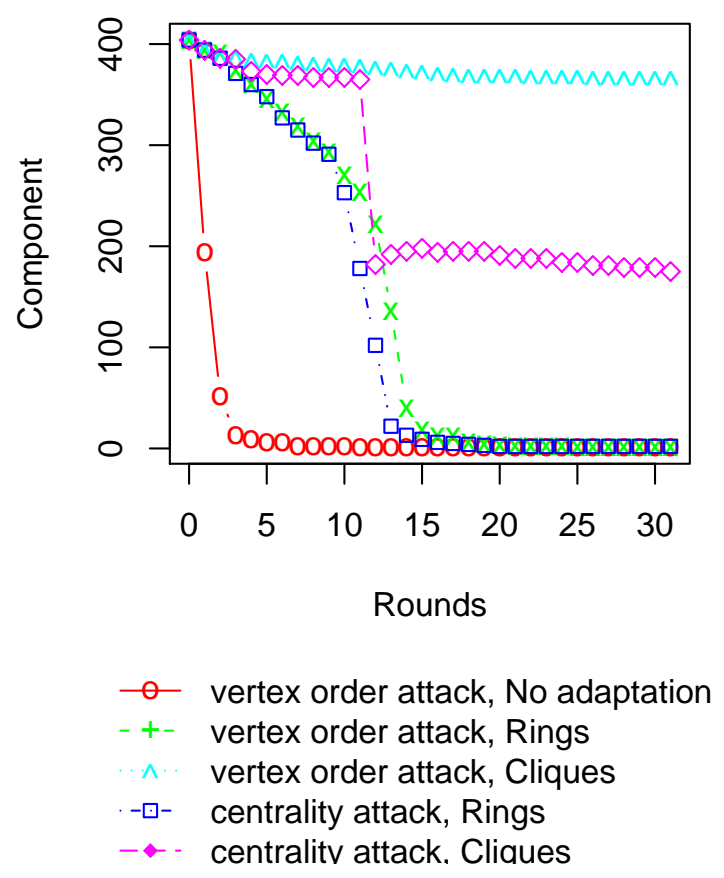

Fig. 3. Rings and Cliques defense under vertex order and centrality attacks 


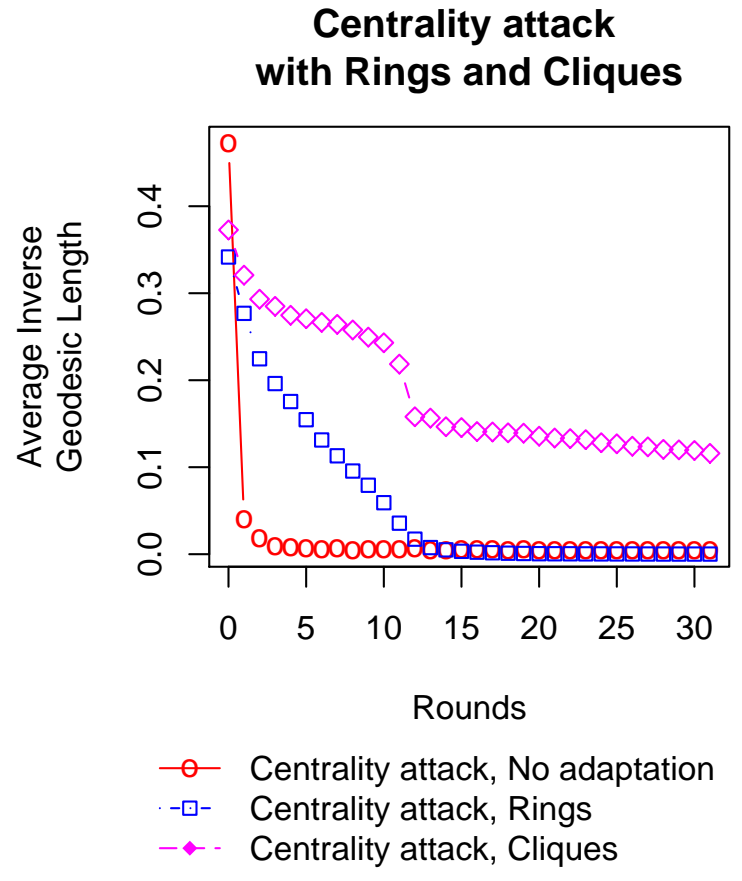

Fig. 4. Average inverse geodesic lengths of rings and clique adaption, under centrality attack
Some insight into the internal mechanics can be gleaned from Figure 4 This shows the average inverse geodesic length. For each node, we find the length of the shortest path to each other node, and take the inverse (we take the length to be infinite, and thus the inverse to be zero, if the nodes are in disjoint components). We average this value over all $n(n-1) / 2$ pairs of nodes. This value falls sharply for defense without adaptation, and falls steadily for defense with rings. These falls reflect increasing difficulty in internode communication. With cliques, the vertex-order attack has little effect, while the centrality attack makes steadily increasing progress on a graph of 400 vertices, until it achieves partition and reduces the largest component to about 200 vertices. But it makes only slow progress thereafter.

\subsection{Clique sizes}

We next ran a simulation comparing how well defense works when using different sizes of rings and cliques. Ring size appears to make little difference; rings are just not an effective defence other than in the very short term. However, varying the clique size yields the results displayed in Figure 5 .

This shows that under a centrality attack, the performance of the defense increases steadily with the size of the clique. There is still a phase transition after about 14 rounds or so after which the largest connected component becomes significantly smaller, but the size of this equilibrium component increases steadily from about 150 with clique size 8 to almost 300 at clique size 20 .

\section{Defence Evolution - Second Round}

Now that we know centrality attacks are powerful, we have tried a number of other possible defences. The most promising at present appears to be a compound defence based on cliques and delegation.

The idea behind delegation is fairly simple. A node that is becoming too well-connected selects one of its neighbours as 

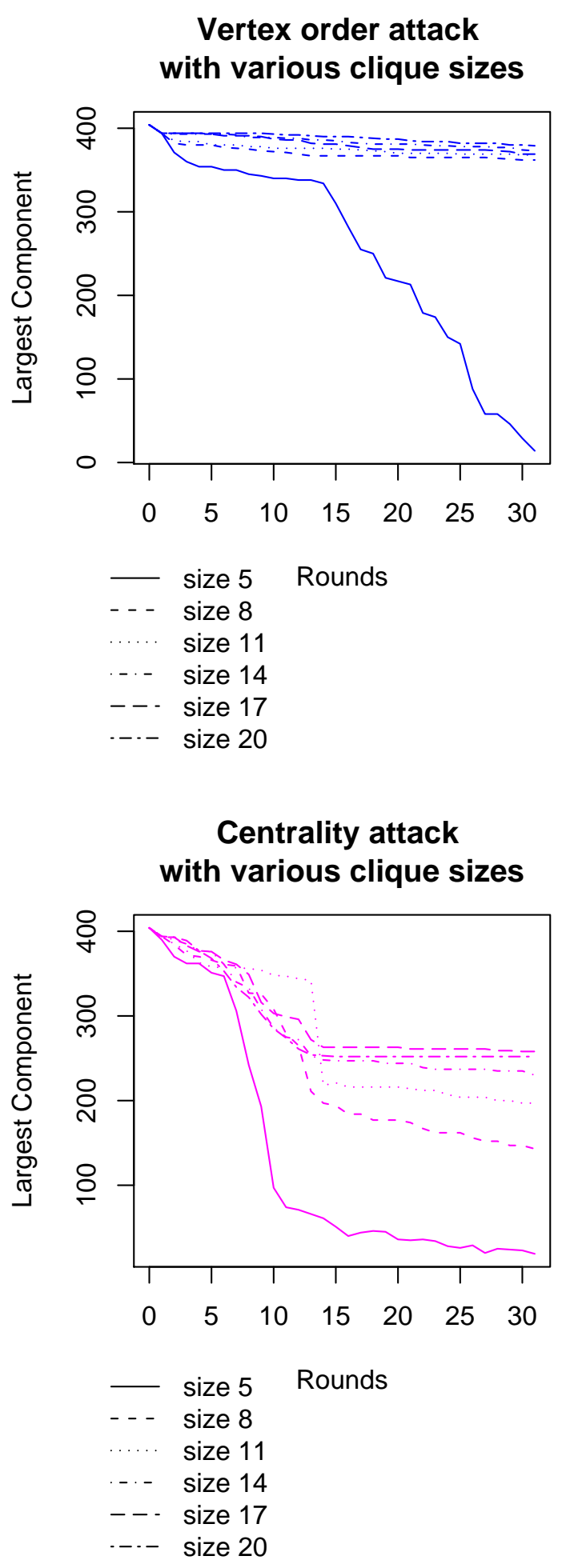

a 'deputy' and connects it to a second neighbour, with which it then disconnects. This reflects normal human behaviour even in peacetime: busy leaders pass new recruits on to colleagues. In wartime, and with an enemy that might resort to vertex-order attacks, the incentive to delegate is even greater. Thus a terrorist leader who gets an offer from a wealthy businessman to finance an attack might simply introduce him to a young militant who wants to carry one out. The leader need now maintain communications with at most one of the two.

Delegation on its own is rather slow; it takes dozens of rounds for delegation to 'immunise' a network against vertex-order attack. If a vanilla scale-free network is going to be exposed to either a vertex-order or centrality attack from the next round, then drastic action (such as clique formation) is needed at once; else it will be disconnected within two or three rounds. Slower defences like delegation can however play a role, provided they are started from network formation or a reasonable time period (say 20 rounds) before the attack begins.

It turns out that the delegation defence, on its own, is rather like the rings of dining steganographers. Network fragmentation is postponed (about 14 rounds with the parameters used here) though not ultimately averted.

What is interesting, however, is this. If we form a network and immunise it by running the delegation strategy, then run a clique defence as well from the initiation of hostilities, this compound strategy works rather better than ordinary cliques. Figure 6 shows the simulation results.

Figure 7 may give some insight into the mechanisms. Delegation results in shorter path lengths under attack: it postpones and slows down the growth of path length that otherwise results from hub elimination. As a result, equilibrium is achieved later, and with a larger minimum connected component.

Fig. 5. Clique recovery with different clique sizes under a centrality attack

\section{Conclusions and Future Work}

In this paper, we have built a bridge between network science and evolutionary game theory. 


\section{Centrality attack with Cliques and Delegation}

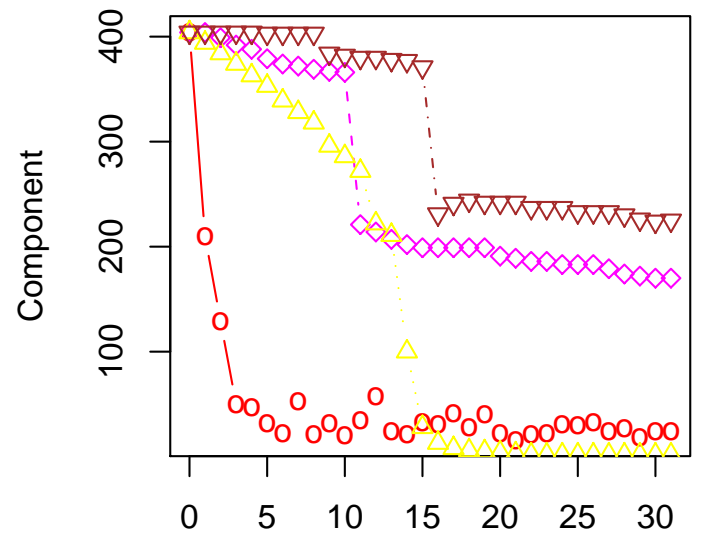

Rounds

$$
\begin{array}{ll}
-0 & \text { No replenishment } \\
-\Delta & \text { Delegation } \\
\diamond & \text { Clique } \\
-\nabla- & \text { Clique + Delegation }
\end{array}
$$

Fig. 6. Component size: clique, immunization by delegation, and combined clique and delegation defenses against centrality attack

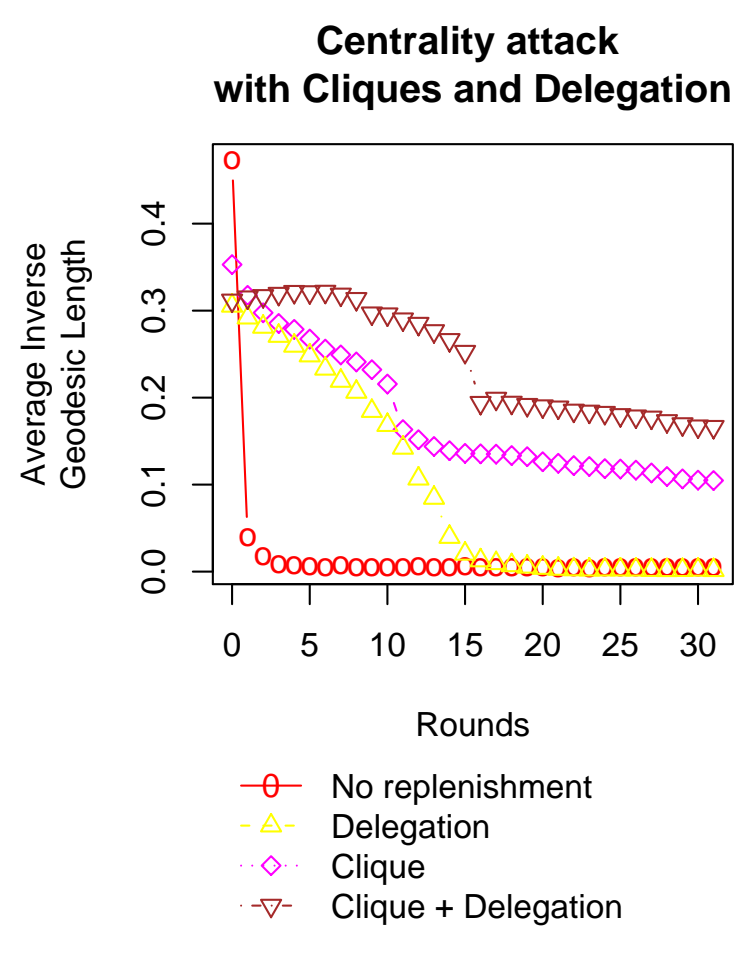

Fig. 7. Clique, immunization by delegation, and combined clique and delegation defenses against centrality attack 
For some years, people have discussed what sort of communications topologies might be ideal for covert communication in the presence of powerful adversaries, and whether network science might be of practical use in covert conflicts - whether to insurgents or to counterinsurgency forces [518. Our work makes a start on dealing with this question systematically.

Albert, Jeong and Barabási showed that although a scalefree network provides better connectivity, this comes at a cost in robustness - an opponent can disconnect a network quickly by concentrating its firepower on well-connected nodes. In this paper, we have asked the logical next questions. What sort of defence should be planned by operators of such a network? And what sort of framework can be developed in which to test successive refinements of attack, defense, counterattack and so on?

First, we have shown that naive defences don't work. Simply replacing dead hubs with new recruits does not slow down the attacker much, regardless of whether link replacement follows a random or scale-free pattern.

Moving from a single-shot game to a repeated game provides a useful framework. It enables concepts of evolutionary game theory to be applied to network problems.

Next, we used the framework to explore two more sophisticated defensive strategies. In one, potentially vulnerable high-order nodes are replaced with rings of nodes, inspired by a standard technique in anonymous communications. In the other, they are replaced by cliques, inspired by the cell structure often used in revolutionary warfare. To our surprise we found that rings were all but useless, while cliques are remarkably effective. This may be part of the reason why cell structures have been widely used by capable insurgent groups.

Next, we searched for attacks that work better against clique defences. We found that the centrality attack of Holme et al does indeed appear to be more powerful, although it can be more difficult to mount as evaluating node centrality involves knowledge of the entire topology of the network. Centrality attacks may reflect the modern reality of counterinsurgency based on pervasive communications intelligence and, in particular, traffic analysis.

Now we are searching for defences that work better against centrality attacks. A promising candidate appears to be the delegation defence, combined with cliques. This combination may in some ways reflect the reported 'virtualisation' strategies of some modern insurgent networks.

Above all, this work provides a systematic way to evolve and test security concepts relating to the topology of networks. Clearly the coevolution of attack and defense can be taken much further. Further work includes testing:

1. networks that grow or shrink, maybe with endogenous replenishment (current recruitment a function of past operational success)

2. imperfectly informed attackers, such as policemen who have access to the records of some but not all phone companies or email service providers, or who must use purely local measures of centrality

3. perfectly informed defenders, who can coordinate connectivity globally

4. budget tradeoffs - for example, a defender might be able to hide specific edges but only at some cost to his replenishment budget

5. heterogeneous networks, with subpopulations having different robustness preferences

6. dynamic strategies that detect opponents' strategies and respond

7. different attacker goals. For example, some say that the Iraqi rebel leader Al-Zarqawi is not bin Laden's subordinate but his competitor. So an attack objective might be not just partition, but to divide the opposition into groups of less than a certain size. When attacking an ad-hoc sensor network, the goal might be to reduce the effective bandwidth, and there might be interaction with routing algorithms.

Preliminary though it is, we suggest that this work has broad potential applicability from making the Internet more resilient against natural disasters and malicious attacks, to the question of how best to disrupt (or design) subversive networks. 
Acknowledgements: We have had useful feedback on an early versionq of this paper from Albert-László Barabási, Mike Bond, George Danezis, Karen Spärck Jones and Chris Lesniewski-Laas. The first author was supported by a scholarship from the Gates Trust.

\section{References}

1. R Albert, AL Barabási, "Statistical Mechanics of Complex Networks", Reviews of Modern Physics 74 (2002) 47

2. R Albert, H Jeong, and AL Barabási, "Error and attack tolerance of complex networks" in Nature v 406 (2000) pp 387-482

3. R Axelrod, "The Evolution of Cooperation" Basic Books, New York (1984)

4. R Axelrod, "The Complexity of Cooperation" Princeton University Press (1997)

5. C Ballester, A Calvó-Armengol and Y Zenou, "Who's Who in Crime Networks - Wanted the Key Player", IUI Working Paper Series 617, The Research Institute of Industrial Economics (2004).

6. AL Barabási, R Albert, "Emergence of scaling in random networks", in Science v 286 (1999) pp 509-512

7. B Bollobás, 'Random Graphs', Academic Press (1985)

8. U Brandes, "A Faster Algorithm for Betweenness Centrality", J. Math. Soc. 25(2) (2001) pp 163177

9. PJ Carrington, J Scott, S Wassermann, 'Models and Methods in Social Network Analysis', Cambridge University Press (2005)

10. D Chaum, "The Dining Cryptographers Problem: Unconditional Sender and Recipient Untraceability", in Journal of Cryptology v 1 (1989) pp 65-75

11. P Erdös, A Renyi, "On Random Graphs", in Publicationes Mathematicae v 6 (1959) pp 290297

12. LC Freeman, "A set of measuring centrality based on betweenness", in Sociometry v 40 (1977) p 35-41

13. MO Jackson, "A Survey of Models of Network Formation: Stability and Efficiency" A, in Group Formation in Economics: Networks, Clubs, and Coalitions, edited by Gabrielle Demange and Myrna Wooders, Cambridge University Press.

14. VE Krebs, "Mapping Networks of Terrorist Cells", in Connections v 12 no 3 ; at http://www.locative.net/tcmreader/index.php?mapping;krebs

15. P Holme, BJ Kim, CN Yoon, and SK Han, "Attack Vulnerability of Complex Networks" in Phys. Rev. E v 65 art. no. 018101, 2002

16. S Milgram, "The Small World Problem", in Psychology Today v 2 (1967) pp 60-87
17. MEJ Newmann, "Structure and Function of Complex Networks", SIAM Review 45 (2003) pp $167-256$

18. MK Sparrow, "The Application of Network Analysis to Criminal Intelligence: An assessment of the prospects", in Social Networks v 13 (1990) pp 253-274

19. N Thompson, "The Network Effect - Why Senegal's bold anti-AIDS program is working", in The Boston Globe January 5, 2003; at http://www.newamerica.net/index.cfm?pg=article\&DocID=1092

20. S Wassermann, K Faust, 'Social Network Analysis', Cambridge University Press (1994)

21. DJ Watts, 'Six Degrees: The Science of a Connected Age', Norton, New York (2003)

22. DJ Watts, SH Strogatz, "Collective Dynamics of Small-World Networks", in Nature v 393 (1998) pp 440-442

23. LA Zhao, KH Park, YC Lai, "Attack vulnerability of scale-free networks due to cascading breakdown", in Physical review E v 70, 035101 (R), 2004 


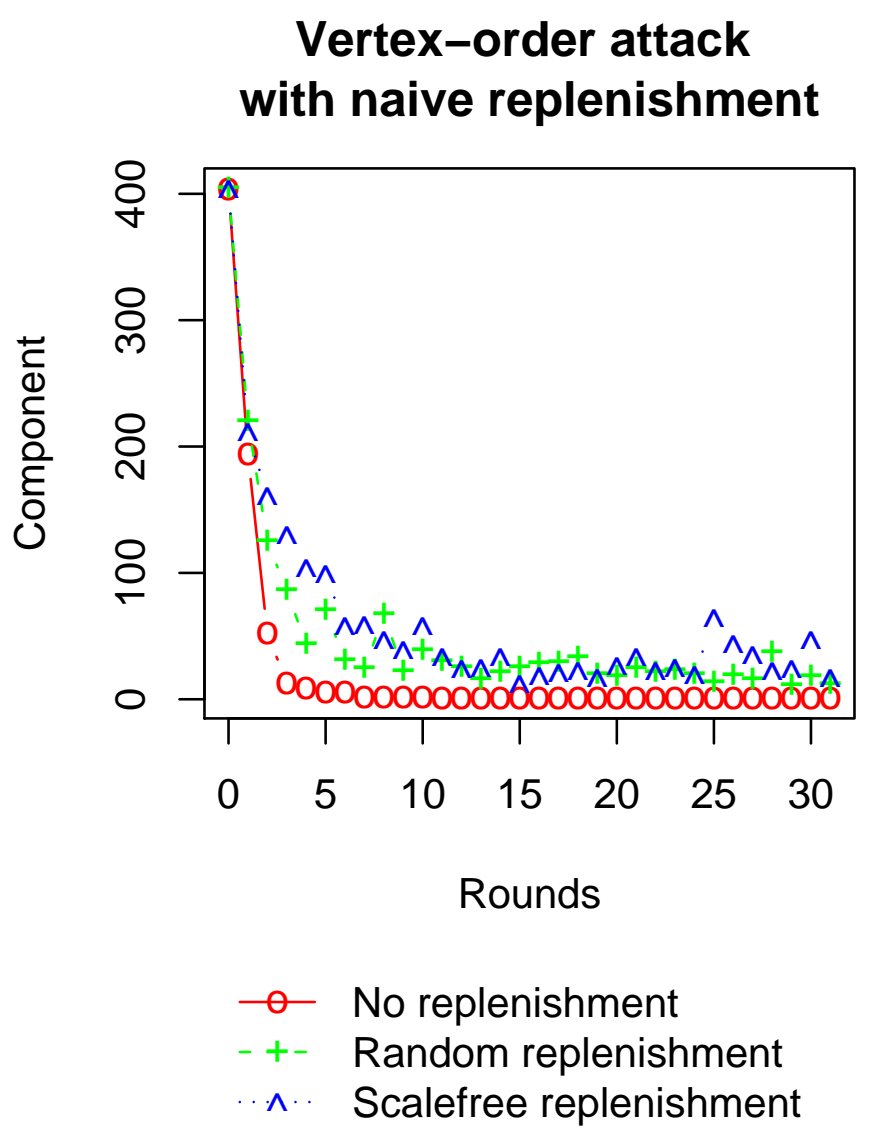

